\title{
The effects of location, orientation, and cumulation of boxes in the Baldwin illusion
}

\author{
ALEXANDER W. PRESSEY and NANCY E. SMITH \\ St. John's College, University of Manitoba, Winnipeg, Canada
}

\begin{abstract}
Three experiments were conducted on variants of the Baldwin illusion. Experiment 1 showed that placing a box on each side of the standard line produced a larger illusion than placing both boxes on the same side of the line. These results failed to support the assimilation theory proposed by Brigell, Uhlarik, and Goldhorn (1977). In Experiment 2, the side of a rectangular box was varied when that side was either parallel or perpendicular to the standard line. The parallel rectangle produced a function that was similar to the one found with the classical Baldwin figure, but the perpendicular boxes produced a monotonically decreasing function with a reversal of illusion evident at the large sizes. The latter function did not support any version of the assimilation theory. The findings of Experiment 3 replicated previous findings that showed that cumulating contours as box size increased had no effect on the illusion. These findings explain two longstanding puzzles about the Müller-Lyer illusion: why a multifinned form is not the sum of its single-finned parts and why the shrinkage form produces a smaller effect than does the expansion form.
\end{abstract}

In the Baldwin configuration, shown in Figure 1, a bisected line is flanked by two squares of unequal size. The two portions of the bisected line do not appear equal. Rather, the part of the line near the small square appears longer than the part near the large square.

Two interpretations of the Baldwin illusion have been proposed. One is based on the notion of contrast, in which differences between stimuli are perceptually enhanced. Thus, the distance near the small square (Distance B in Figure 1), which is a relatively large length, is judged in the context of the width of the small box, which is a relatively small length, and because of contrast, a phenomenal elongation of Distance B occurs (Baldwin, 1895; Restle \& Merryman, 1969). A second interpretation is based on the concept of assimilation, in which differences between stimuli are perceptually reduced. Distance B in Figure 1 is judged in the context of the distances between the bisecting mark and the outer edge of the box. Because these distances are all longer than the standard distance, the standard assimilates to (or averages with) the longer lengths and itself appears elongated. The interpretation based on assimilation is favored by Brigell, Uhlarik, and Goldhorn (1977), by Clavadetscher and Anderson (1977), and by Pressey and Wilson (1980). Indeed, the quantitative version of Pressey's assimilation theory has been found to provide a good fit to the function relating illusion to box size, except for the cases in which the boxes were so large that a reversal of the illusion occurred (Pressey \& Wilson, 1980).

This research was supported by a grant from the Natural Sciences and Engineering Research Council of Canada. Requests for reprints should be sent to Alexander Pressey, St. John's College, University of Manitoba, Winnipeg, Canada R3T 2M5.

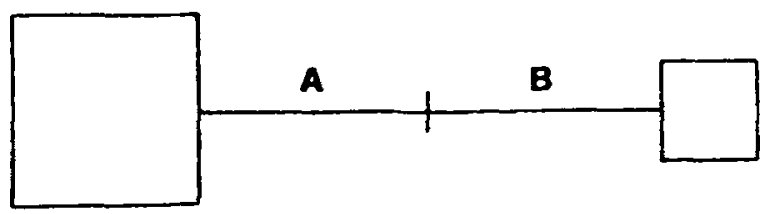

Figure 1. The Baldwin illusion. Distance $A$ appears shorter than Distance B.

\section{EXPERIMENT 1}

Brigell et al. (1977) argued that the critical causal variable in many illusions of length is the framing ratio, that is, the ratio of the length of the entire figure to the length of the standard line. Their explanation of the inverted- $U$ function that relates illusion to box size was based on a neurophysiological model. According to Brigell et al.,

viewing a stimulus activates length-selective units in proportion to their sensitivities to that length. Each unit responds to only a limited range of proximal stimulus lengths. The maximal overestimation of focal shaft length should occur when the same unit is most sensitive to the total figure length and also is the rightmost unit in the distribution produced by the focal shaft when the shaft is presented alone. (p. 116)

Pressey's assimilation theory (e.g., Pressey \& DiLollo, 1978) also focuses on the size of the contextual stimulus, which he calls the range variable. The increasing portion of the inverted- $U$ function is explained by the fact that the average of the focal and contextual stimuli increases as the size of the contextual stimulus (frame) increases. The decreasing portion is explained by the fact that the probability of averaging decreases, because (1) the 
spatial separation between focal and contextual stimuli increases and (2) the probability of attending to the contextual stimulus decreases.

The purpose of this study was to provide a test of these two theories of the Baldwin illusion by varying the location, but not the ratio (or the range), of the contextual magnitude relative to the standard magnitude. The theory proposed by Brigell et al. (1977) would predict that location would have no effect on the illusion, whereas Pressey's theory would contend that location would have a profound effect on the size of the illusion.

Location was varied by placing a box on each side of a standard line in one condition and then placing both boxes on one side in a second condition (see Figure 2). Figure 2 also illustrates a third configuration, which was identical to the second except that the vertical bisecting line was removed. This configuration was included because Pressey's theory suggests that the number of contours could be an important variable in determining the contextual length, and hence the size of the illusion.

\section{Method}

Subjects. The subjects were 40 right-handed men and women who participated as part of a course requirement. We asked that only those with good vision, with or without glasses, volunteer.

Materials. Each target was drawn with black ink on white 21.6 $\times 27.9 \mathrm{~cm}$ sheets of paper. All standard lines were $50 \mathrm{~mm}$ long and $.25 \mathrm{~mm}$ wide.

In the targets with two flanking boxes, the sides of the boxes were $1.25,2.5,3.75,5,10,15,20,30,40$, and $50 \mathrm{~mm}$ long. In the targets with the two boxes placed on one side, the lengths were $2.5,5,7.5,10,20,30,40,60,80$, and $100 \mathrm{~mm}$. The third set of targets was the same as the second set except that the vertical bisecting line was removed.

All standard lines were drawn parallel to the long side and in the center of the sheet. In addition, because the method of production was employed (Pressey, 1974b), different starting positions were used for each target. In one case, a small black dot was drawn $80 \mathrm{~mm}$ below and $8 \mathrm{~mm}$ to the left of the left edge of the standard line, and in the second case, the dot was drawn $80 \mathrm{~mm}$ below and $8 \mathrm{~mm}$ to the right of the left edge of the starting line. Control lines with no flanking boxes were also employed.

High-quality and high-resolution printing methods were employed to reproduce the targets. The targets were presented on a white wooden holder that was $39.5 \mathrm{~cm}$ high and $37 \mathrm{~cm}$ wide and was tilted backward at an angle of $20^{\circ}$. An adjustable chinrest was located directly in front of the holder so that the eyes were perpendicular to the target at a distance of about $41 \mathrm{~cm}$.

Design. A $3 \times 10$ within-subjects design was employed in which there were 3 types of configurations and 10 levels of box size. In addition, two balancing variables-starting position and left-right box positions-formed a between-subjects replication matrix. The subjects were randomly assigned to one of the four replication cells and proceeded through the 10 levels of box size and the 3 types of configurations according to the dictates of their particular cell.

Procedure. A stimulus packet was compiled for each subject. It consisted of one target from each of the combinations of 10 box sizes and 3 types of configurations, preceded by four control targets at the beginning of the series. An additional four control targets were given at the end of the series.

The subject was seated in front of the target holder with his/her chin in the rest. A sample target was shown and the subject was asked to draw, freehand, a line that appeared equal to the standard line. The subject was told to make judgments carefully but not to spend too much time on any target. When the subject understood the task, testing proceeded.

All targets were centered on the holder and were presented manually by the experimenter. The rate of presentation was roughly constant; that is, approximately $3 \mathrm{sec}$ were taken to remove and replace a target.

\section{Results and Discussion}

Measurements of the produced line were made with a straightedge scale and were accurate to $0.5 \mathrm{~mm}$. For each subject, the mean of the eight control lines was subtracted from each of the responses to the experimental targets and these scores were averaged across subjects. The results are shown in Figure 2, in which box size is expressed as a ratio of the total contextual length to the standard length.

Analysis of variance showed that the variable of box size was significant $[F(9,390)=3.53, p<.01]$ and the variable of configuration was significant $[F(2,77)=9.19$, $p<.01]$, but the interaction between box size and configuration was not significant $[F(18,777)=1.49$, $p>.05]$.

The most striking finding is that the symmetrical location of boxes on both sides of the standard line produced a substantially greater illusion than did the location of both boxes on one side of the standard line. These results do not conform to the framing theory of Brigell et al. (1977); according to that theory, the ratios of frame to standard are the same in all cases, and therefore the illusion should be of the same magnitude.

Pressey's assimilation theory, on the other hand, does predict the present results. When a box is moved from one side and joined with the box on the other side, that box is now farther from the standard line than it was originally. Because of this increase in spatial separation, there will be a decrease in the probability that averaging of the box with the standard line will occur. In addition, the box that is moved will be located farther away from the center of attention (Pressey \& Bross, 1973), which will also reduce the probability that averaging will occur. Thus, because of an increase in discriminability between box and line and because of a decrease in the salience of the box, the asymmetrically located boxes will be less effective than the symmetrically placed boxes in altering the apparent size of the standard line.

Analysis revealed no significant differences between the two conditions in which boxes were asymmetrically located. That is, removal of the interior vertical line, which created a single rectangular box, did not affect the magnitude of distortion. This result is damaging to both Brigell's and Pressey's versions of assimilation theory. For example, in making quantitative predictions of the Ponzo illusion (Pressey, Butchard, \& Scrivner, 1971) it is implied that all features of the contextual stimuli have the potential to alter the percept. The same implication seems to be contained in the neurophysiological theory proposed by Brigell et al. (1977). Thus, both theories would predict that the interior edge would affect the illu- 


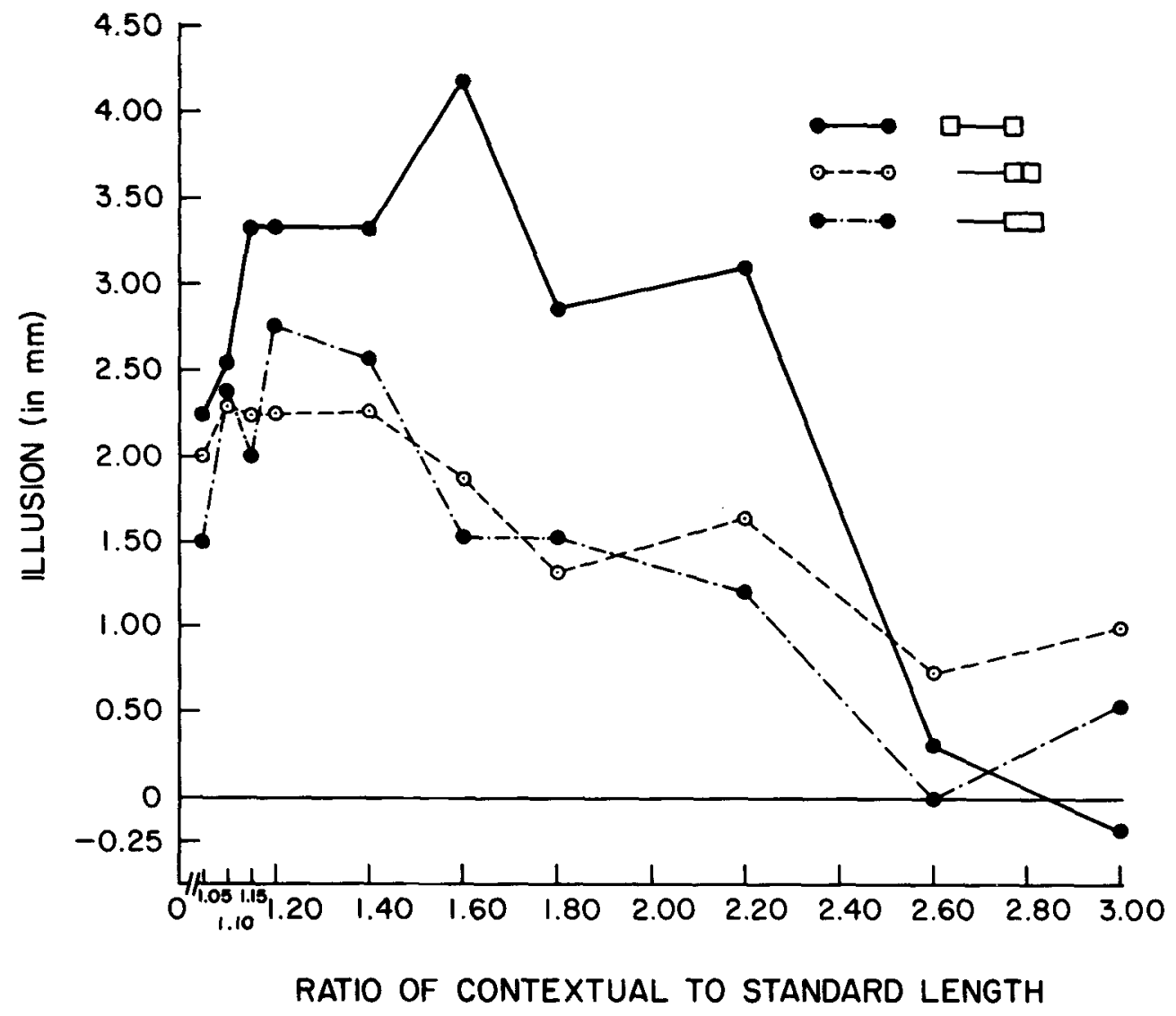

Figure 2. Effects of box size on the magnitude of the Baldwin illusion when boxes are symmetrically and asymmetrically located.

sion, and the failure to find a difference is a puzzle for both theories. This problem was pursued separately (Experiment 3).

A final point of interest is that no reversal of illusion occurred at very large ratios of box size to focal line size. This result contradicts data from many subjects tested in our laboratory (e.g., Pressey \& Wilson, 1980; Experiment 3 of the present study), who exhibited reliable reversals in direction of distortion at very large box sizes. But the results of this experiment are consistent with those obtained by Brigell et al. (1977) and by Clavadetscher and Anderson (1977), who also found no reversals. The most reasonable explanation at this time is that the reversal depends upon the psychophysical method and seems to be limited to the method of bisection. When the methods of reproduction, adjustment, or selection are employed, no reversal effects are observed.

\section{EXPERIMENT 2}

Most investigations of the Baldwin illusion have employed square boxes to alter the apparent size of a standard line. But a square has at least three attributes (height, width, and area) that might contribute to the illusion. The main objective of this study was to manipulate the orientation of the contextual features in relation to the stan- dard line in order to determine which attribute is primarily responsible for causing distortion.

The assimilation theories proposed by Brigell et al. (1977) and Pressey (e.g., Pressey \& DiLollo, 1978) contend that the critical feature is the attribute that is parallel to the orientation of the standard. Thus, in Figure 3, for example, both Brigell et al. and Pressey would argue that it is the width of the box that is the sole determiner of distortion. Brigell et al. (1977, p. 110) state that it is the ratio of total figure length to shaft length that determines the Baldwin illusion, and Pressey uses (implicit or explicit) contours that are parallel to the standard contour to de-

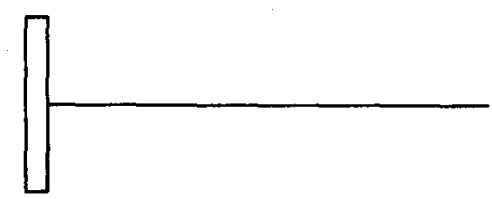

Figure 3. Parallel (A) and perpendicular (B) orientation of boxes in the Baldwin configuration. 
rive quantitative estimates of distortion. Neither assimilation theory predicts any substantial effect of changing box size in an orthogonal manner, as shown in Figure 3B.

\section{Method}

Subjects. Thirty-three men and women in an undergraduate research methods course participated. None reported being familiar with the Baldwin illusion. Two subjects were eliminated because they failed to understand instructions and one was eliminated because she reported having defective vision.

Materials. Twenty targets, similar to those shown in Figure 3, were drawn with black ink and centered on $28 \times 21.7 \mathrm{~cm}$ sheets of white paper. The shaft was a solid 100 -mm line drawn parallel to the long side of the sheet. The long sides of the rectangles were $2.5,5,7.5,10,20,30,40,50,80$, and $100 \mathrm{~mm}$, and the short side was a constant $2.5 \mathrm{~mm}$. The width of each contour was approximately $0.5 \mathrm{~mm}$. Targets were reproduced by high-fidelity printing.

Design. A $2 \times 10$ mixed design was employed in which the orientation of the rectangles was the between-subjects variable and the long side of the rectangle was the within-subjects variable.

For each subject a booklet of 10 pairs of targets in random order was constructed. On half of the trials the box was located on the left of the shaft and on the other half it was located on the right of the shaft. The position of the box was balanced on adjacent trials and the initial position was alternated between subjects.

Procedure. The subjects were seated at a table with the package of targets lying face down and to one side of them. A sample target was crudely drawn on a chalkboard and it was explained that the task was to draw a sharp but well-defined mark that appeared to bisect the horizontal line. It was emphasized that only the visual modality could be used and that the subjects were to draw the mark where it seemed to bisect the line and not where they thought it "really" bisected it. When the experimenter was satisfied that all subjects (who were tested in a single group) understood the task, they proceeded by removing the top sheet, marking it, and then placing it face down on the opposite side of the original pile.

\section{Results and Discussion}

The position of the bisecting mark was measured to the nearest $0.5 \mathrm{~mm}$. If the mark was displaced toward the box, a positive value was assigned; this indicated distortion in the expected direction. That is, a mark that was objectively centered would make the distance between box and mark appear too large, with the result that the mark would have to be moved toward the box to equate subjectively the two portions of the shaft. Of course a negative value indicated that the distance between the box and the objective center appeared too short.

The mean scores, plotted as a joint function of orientation and size of box, are shown in Figure 4. Analyses of variance indicated that all three variables were significant [for orientation, $F(1,139)=137.2, p<.01$; for box size, $F(9,140)=4.44, p<.01$; for the interaction, $F(9,139)=2.94, p<.01]$.

These data clearly indicate that the orientation of the contextual box had a profound effect on the Baldwin illusion. As such, they are in line with an assimilation theory that contends that the side of the box that is parallel

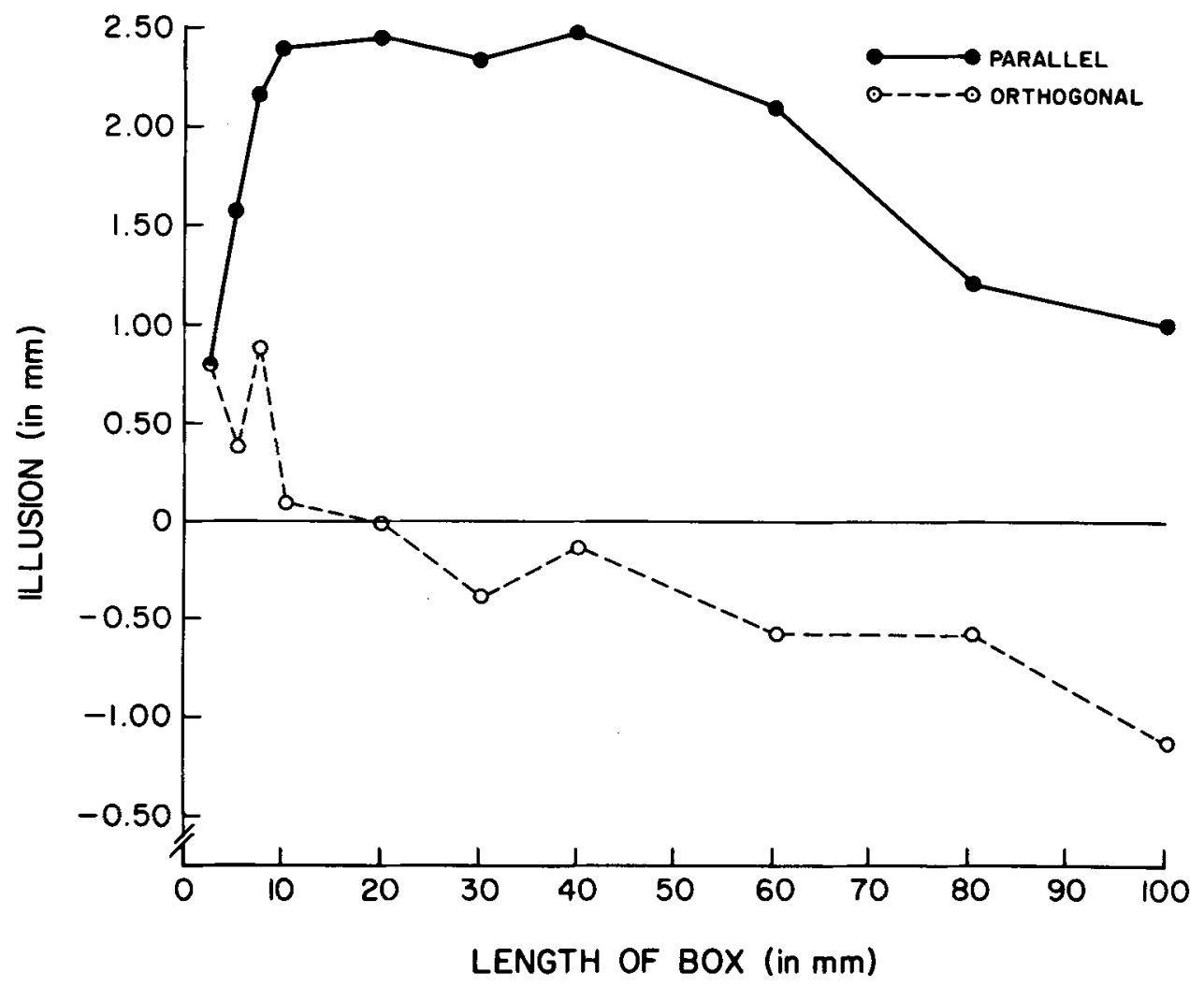

Figure 4. The Baldwin illusion as a joint function of box size and relative orientation. 
to the standard line is of primary importance in producing distortion.

Yet it is clear that an assimilation theory is not sufficient to explain the results shown in Figure 4. Increasing the side of the box that is perpendicular to the standard line resulted in a decline in the traditional form of the illusion $[F(9,139)=4.08, p<.01]$ and produced a reversed effect when the rectangles were very long. (For example, the value of $t$ for the $100-\mathrm{mm}$ box was 2.75 , $p<.05$.) Since assimilation theories require that there be a difference in the size of the contextual magnitude in order to produce a difference in distortion, and since increasing the side of the box that is perpendicular to the standard line produces no such change, predictions from assimilation theories are not validated. This failure is similar to the failure of assimilation theories to predict data from an $\mathrm{H}$ figure (Adam \& Bateman, 1980; Restle \& Decker, 1977) when the wings of that figure are increased. Clearly, there is some factor linked to the relative orientation of the standard and contextual features that has not been isolated.

Previous research in which relative orientation was manipulated may have some bearing on the present findings. For example, Fry and Bartley (1935) concluded that parallel contours inhibit each other and that perpendicular contours can result in facilitation. In addition, Sayons and Prysiazniuk (1963) used a frame that continually lengthened and shortened around a stationary line. They induced alpha motion in the line both when the frame was co-oriented with the line and when it was perpendicular to the line, but the effect was greater in the former case. These studies reinforce the importance of relative orientation but give no clue about the exact manner in which such a variable mediates distortion.

\section{EXPERIMENT 3}

Results from Experiment 1 and from a previous study (Pressey, 1985) indicated that the presence of an internal contour within the contextual box appeared to have no effect on the magnitude of illusion. This finding is important for any theory of illusions, but it is particularly important for an assimilation theory. In Pressey's theory, for example, all contours have the potential to form a context, and they do so depending upon where they are located in relation to the standard line and in relation to the center of the attentional field. Thus, if one were to cumulate prior boxes as the variable of box size was increased (as shown in Figure 5B), Pressey's theory would predict that the illusion would be the average of the contribution of all boxes. The function for cumulated box size would be much flatter than the function produced by outline boxes. However, existing data do not support this prediction. The functions relating box size to illusion were not significantly different for cumulated and outline boxes (Pressey, 1985). However, this conclusion was based on data obtained from two experiments which, for some reason, might not have been comparable. The purpose of the

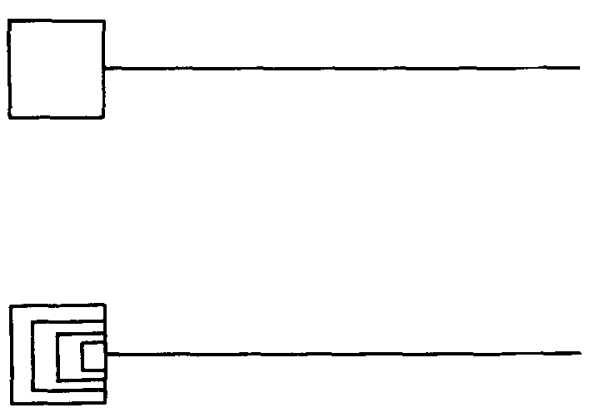

A

Figure 5. Examples of outline and cumulated boxes in the Baldwin target.

present experiment was to compare the effects of outline and cumulated boxes on the Baldwin illusion in a single experiment. In the cumulated box condition, each succeeding box contained all preceding boxes, so that the largest box contained the entire range of boxes employed in the study.

\section{Method}

Subjects. Eighty men and women enrolled in introductory psychology courses participated as part of a course requirement. Only those who reported having good vision with or without glasses were tested.

Materials. Twenty targets, half of which consisted of outline boxes and the other half of cumulated boxes similar to those shown in Figure 5, were drawn with black ink on $28 \times 21.7 \mathrm{~cm}$ sheets of white paper. The shaft was a solid $100-\mathrm{mm}$ line drawn parallel to the long side of the sheet. The sides of the square boxes were the same as the long sides of the rectangles used in Experiment 2. The width of each contour was approximately $0.5 \mathrm{~mm}$. Targets were reproduced by high-fidelity printing.

Design. A $2 \times 10$ mixed design was employed in which type of box (outline or cumulated) was the between-subjects variable and the size of the largest box was the within-subjects variable.

For each subject a booklet was constructed of 10 pairs of targets arranged in a unique random order. Sheets of heavy blue paper were inserted between the targets and the package was stapled in the upper left corner. On half of the trials the box was located on the left of the shaft and on the other half it was located on the right of the shaft. The position of the box was balanced on adjacent trials and the initial position was alternated between subjects.

Procedure. The subjects were tested in four sessions in a manner similar to that described in Experiment 2. The only difference was that targets were retained in a single package during testing.

\section{Results}

Scores were obtained for each target in the manner described in Experiment 2. For each subject a single measure of illusion was obtained by averaging scores from the left and right box positions. The results for the two groups are shown in Figure 6. Analysis of variance indicated that the only significant variable was size of box $[F(9,390)=34.3, p<.01]$. The $F$ values for the remaining variables were less than 1.0.

These results are in complete agreement with previous findings (Pressey, 1985) which showed that a box containing several smaller boxes produced an illusion that was equivalent to the one produced by the largest box alone. 


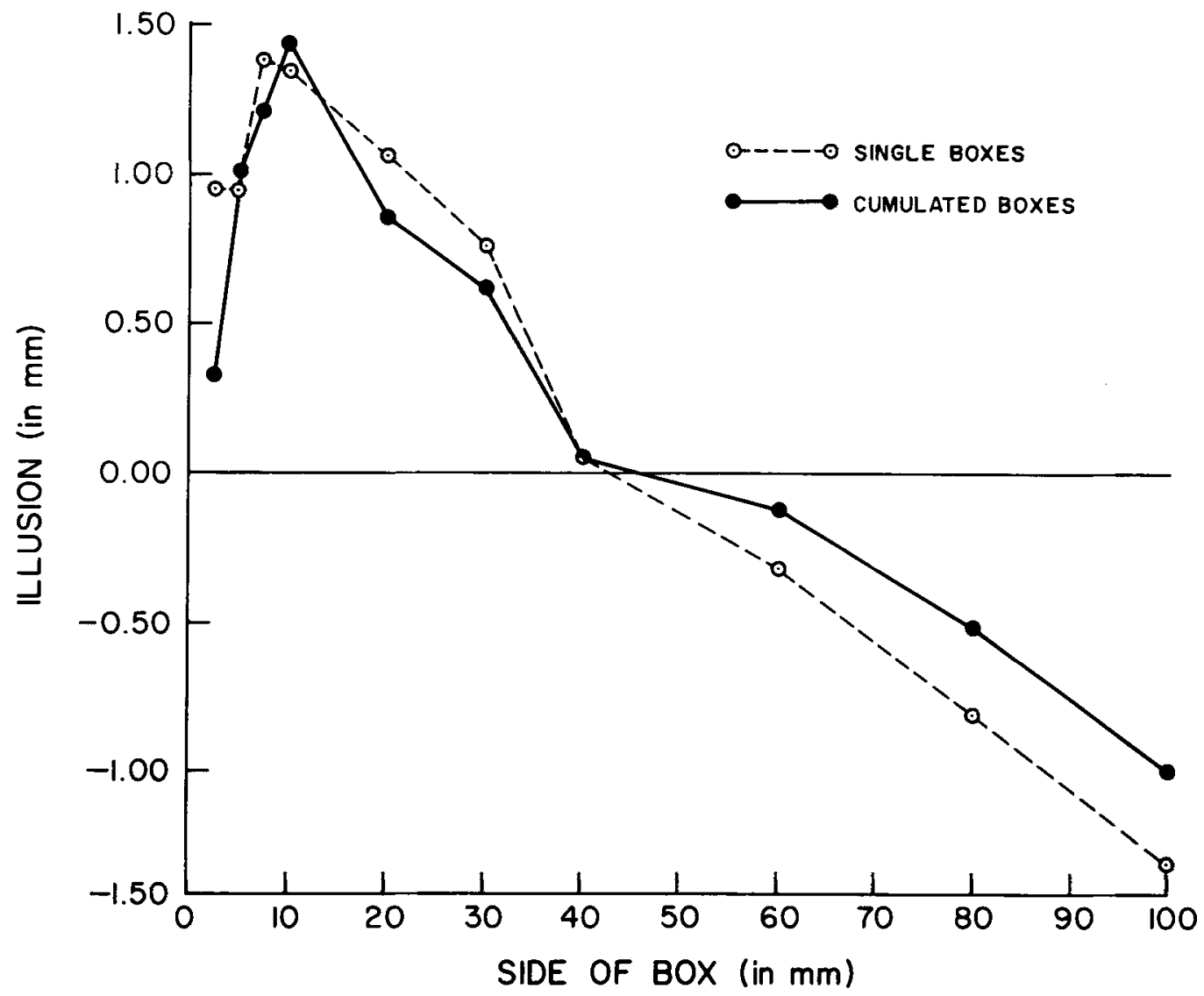

Figure 6. The Baldwin illusion as a joint function of form and size of box.

\section{Discussion}

These results suggest that in judging the standard magnitude in the Baldwin figure, the individual inadvertently processes the external edge of the contextual box but not the edges embedded within the box. Why such selectivity occurs is not clear, but the fact that it does provides an explanation for at least two persistent problems in the area of visual illusions.

The first involves the discovery that the effect of two different angled fins in the Müller-Lyer illusion (see Figure 7) is not simply the average of the illusions that are exhibited when the fins are presented separately (Pressey \& Wilson, 1977). Instead, in the expansion form, the multifinned effect tends to be larger than the average, whereas, in the contraction form, the multifinned effect tends to be smaller than the average of the effects of the two fins. Pressey and Wilson suggested that this nonadditivity is due to the presence of a general "elongation effect" caused by the presence of a filled-space illusion, or perhaps by a judgment involving numerosity. The results of the present experiment suggest an alternative interpretation. If internal contours are disregarded by the perceptual system, then it is the outermost fin that will produce the greatest effect in the multifinned illusion. This means that the shrinkage form of the multifinned target will produce an illusion equivalent to that produced by a target with a single ingoing fin containing a large in- terior angle; that is, it will produce an illusion that is smaller than the average of the illusions produced by the two fins presented separately. On the other hand, in the expansion form of the multifinned illusion, the outermost fin will be the acute angled fin, which means that it will behave like the acute angled fin presented alone and thus will produce an illusion that is larger than the average of the illusions produced by the two fins presented separately.

The second problem that may be resolved by the findings in Experiment 3 concerns the asymmetry between the shrinkage and expansion forms of illusions such as the Müller-Lyer, the Delboeuf, and the Ebbinghaus (Titchener) circles (e.g., Bayer \& Pressey, 1972; Binet, 1985; Greist-Bousquet \& Schiffman, 1981; Ogasawara, 1952; Pressey, 1977). The most common finding is that the shrinkage effect is smaller than the expansion effect. Such a result is readily explained by the fact that it is the

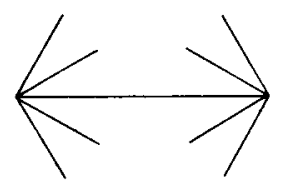

A

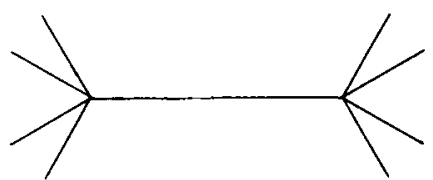

B
Figure 7. Multifinned versions of the Müller-Lyer illusion. 
outermost contour that is most heavily weighted in a percept. For example, in the shrinkage form of the MüllerLyer pattern, it is the ends of the line that form the outermost contours, whereas in the expansion form it is the ends of the fins that form the outermost contours. Thus, in the former case the edges of the contour correspond to the stimuli that are to be judged, with the result that distortion is minimal. However, in the latter case the outermost contours do not correspond to the stimuli that are to be judged, and the result is a large distortion of the judged length.

If this is the correct interpretation of asymmetry in the two forms of the Müller-Lyer illusion, then the argument that the two forms are fundamentally different illusions (Day \& Dickinson, 1976; Greist-Bousquet \& Schiffman, 1981; Sekuler \& Erlebacher, 1971) may not be valid. The correct interpretation may well be that both forms of distortion are caused by the size of the contextual stimuli, but that the different magnitudes of distortion are due to the different spatial locations of the contextual stimuli. It is well established that the spatial location of the contextual stimuli, in relation to both the focal and the comparison stimuli, has a large effect on the magnitude of illusion (e.g., Pressey, 1974a; Pressey \& Murray, 1976; Pressey, Wilson, \& Harper, 1980), and it seems that the asymmetry of the two forms of the Müller-Lyer illusion (and others) is simply a manifestation of that more general principle.

\section{GENERAL DISCUSSION}

The results of the present experiments on the Baldwin illusion indicate that although an assimilation theory can account for many of the robust features of the data, several more subtle features are not so readily explained. In particular, contours that are perpendicular to the standard line produce effects entirely different from those produced by parallel contours, and no assimilation theory, as currently formulated, can predict such effects. In addition, the failure of contours located within the contextual box to affect distortion was not anticipated by assimilation theories. Just how damaging these failures are for an assimilation theory is difficult to assess. Several investigators (e.g., Coren \& Girgus, 1978; McClellan, Bernstein, \& Garbin, 1984) have argued that such a theory is based on the notion of critical stimulus, but that within- and between-subjects differences in cue utilization make it impossible to isolate such a stimulus. This is certainly a plausible argument, but it does challenge one of the fundamental tenets of theory construction, namely, that parsimony should rule.

\section{REFERENCES}

ADAM, J., \& BATEMAN, L. (1980). Control stimuli in investigations of the acute-angled and obtuse-angled Müller-Lyer illlusions. Perception, 9, 467-474.

BALDWIN, J. M. (1895). The effect of size-contrast upon judgments of position in the retinal field. Psychological Review, 2, 244-259.

BAYer, C. A., \& Pressey, A. W. (1972). Geometric illusions as a func- tion of pigmentation of the Fundus oculi and target size. Psychonomic Science, 26, 77-79.

BINET, A. (1985). La mesure des illusions visuelles des les enfants. Revue Philosophique, 40, 11-25.

Brigell, M., Uhlarik, J., \& Goldhorn, P. (1977). Contextual influences and illusions of extent. Journal of Experimental Psychology: Human Perception \& Performance, 3, 105-118.

Clavadetscher, J. E., \& Anderson, N. H. (1977). Comparative judgment: A test of two theories using the Baldwin figure. Journal of Experimental Psychology: Human Perception \& Performance, 3, 119-135.

Coren, S., \& GrRgus, J. S. (1978). Seeing is deceiving: The psychology of visual illusions. Hillsdale, NJ: Erlbaum.

DAY, R. H., \& Dickinson, R. G. (1976). Apparent length of the arms of acute and obtuse angles, and the components of the Müller-Lyer illusion. Australian Journal of Psychology, 28, 137-148.

FrY, G. A., \& BARTLEY, S. H. (1935). The effect of one border in the visual field upon the threshold of another. American Journal of Physiology, 112, 414-421.

Greist-Bousquet, S., \& Schiffman, H. R. (1981). The many illusions of the Müller-Lyer: Comparisons of the wings-in and wingsout illusions and manipulation of standard and dot forms. Perception, 10, 147-154.

McClellan, P. G., Bernstein, I. H., \& Garbin, C. P. (1984). What makes the Mueller a liar: A multiple-cue approach. Perception \& Psychophysics, 36, 234-244.

Ogasawara, J. (1952). Displacement-effect of concentric circles: Japanese Journal of Psychology, 22, 224-234.

Pressey, A. W. (1974a). Evidence for the role of attentive fields in the perception of illusions. Quarterly Journal of Experimental Psychology, 26, 464-471.

Pressey, A. W. (1974b). Measuring the Ponzo illusion with the method of production. Behavior Research Methods \& Instrumentation, 6, $424-426$.

Pressey, A. W. (1977). Measuring the Titchener circles and Delboeuf illusions with the method of adjustment. Bulletin of the Psychonomic Society. 10, 118-120.

Pressey, A. W. (1985). The role of internal contextual features in the Baldwin illusion. In J. L. McGaugh (Ed.), Contemporary psychology: Biological processes and theoretical issues. New York: Elsevier.

Pressey, A. W., \& Bross, M. (1973). Assimilation theory and the reversed Müller-Lyer illusion. Perception, 2, 211-217.

Pressey, A. W., Butchard, N., \& Scrivner, L. (1971). Assimilation theory and the Ponzo illusion: Quantitative predictions. Canadian Journal of Psychology, 25, 486-497.

Pressey, A. W., \& DiLollo, V. (1978). Effects of distance between standard and comparison lines on the Müller-Lyer illusion. Perception \& Psychophysics, 24, 415-419.

Pressey, A. W., \& Murray, R. (1976). Further developments in the assimilation theory of geometric illusions: The adjacency principle. Perception \& Psychophysics, 19, 536-544.

Pressey, A. W., \&iLson, A. E. (1977). The hydra-headed MuellerLyer effect: A theoretical puzzle. Perception \& Psychophysics, 22, 392-394.

Pressey, A. W., \& Wilson, A. E. (1980). Assimilation theory and the Baldwin illusion. The Italian Journal of Psychology, 7, 65-73.

Pressey, A. W., Wilson, A. E., \& HARPER, D. (1980). Evidence for the role of attentive fields in masking. Perception, 9, 31-36.

Restle, F., \& Decker, J. (1977). Size of the Mueller-Lyer illusion as a function of its dimensions: Theory and data. Perception \& Psychophysics, 21, 489-503.

Restle, F., Merryman, C. (1969). Distance and an illusion of length of line. Journal of Experimental Psychology, 81, 297-302.

Sayons, K., \& Prysiazniuk, A. W. (1963). Kinetic frame effects: I. Alpha motion. Perceptual \& Motor Skills, 16, 581-584.

SekUler, R., \& ERLEBACHER, A. (1971). Two illusions of Muller-Lyer: Confusion theory reexamined. American Journal of Psychology, 84, $477-486$.

(Manuscript received January 22, 1986; revision accepted for publication June 4, 1986.) 